\title{
Background Modelling in transmission X-Ray Powder Diffraction (XRPD) of Pharmaceutical Compounds: a comparative analysis of different approaches
}

$\underline{\text { Bárbara A. Ramírez Almaguer }}{ }^{* 1}$, Mathilde Reinle-Schmitt², Mickael Morin², Lauro Bucio ${ }^{1}$, Antonio Cervellino ${ }^{3}$, Fabia Gozzo ${ }^{2}$

\author{
${ }^{1}$ Instituto de Física, Universidad Nacional Autónoma de México, México, D.F. \\ 2 Excelsus Structural Solutions, PARK INNOVAARE, Villigen, Switzerland. \\ 3 Paul Scherrer Institute, Villigen Switzerland. \\ email: fabia.gozzo@excels.us
}

Pharmaceutical solids can exist in several forms: polymorphic crystalline and amorphous arrangements. Tablets are solids preparations of active pharmaceutical ingredients and additives; many of the additives commonly used are poorly crystalline or amorphous, determining the final solubility in the drug. The ability of correctly differentiate the extrinsic (non-sample dependent) and intrinsic (sample dependent) contributions to the background is a crucial step both for the quantification of non-crystalline phases in Quantitative Phase Analysis (QPA) and Pair Distribution Function (PDF) analysis of active pharmaceutical ingredients, excipients, and formulations. Pair Distribution Function method additionally requires the subtraction of incoherent and inelastic scattering.

Different approaches have been developed in the last years to model these extrinsic contributions; in this work, three models are reviewed and compared.

Riello ${ }^{1}$ and Ottani ${ }^{1}$ (1995) model: background modelled taking into consideration the air scattering, incoherent scattering and average diffuse scattering due to the thermal disorder. This model is developed for Bragg Brentano geometry.

PathCap (2012): developed at the Swiss Light Source Powder Station (Cervellino², 2012) this model removes capillary and air scattering contribution, performing ray tracing absorption corrections assisted by experimental linear absorption coefficient data.

Parametrical Approach (2019); developed by Excelsus Structural Solutions (Gozzo $\left.{ }^{3}, 2019\right)$ this method parametrically optimizes parameters in relatively large sets of high-resolution synchrotron XRPD data collected under the same experimental conditions on samples only differing by the powder packing density. Nominal packing density values are checked against experimental linear absorption coefficient values and iteratively refined. The Parametrical Approach model takes inelastic scattering, compositions, and nominal and experimental packing density into account.

A comparison among the three methods is performed for two crystalline materials, $\mathrm{Al}_{2} \mathrm{O}_{3}$ (characterized by negligible diffuse scattering but not negligible absorption at the working photon energy) and crystalline paracetamol (characterized by small absorption but undefined diffused scattering) to assess the applicability of these methods to pharmaceuticals as well as the accuracy that can be reached.

\footnotetext{
${ }^{1}$ Riello P, Fagherazzi G, Clemente D, Canton P (1995) X-Ray Rietveld analysis with a physically based background. J Appl Crystallogr 28:115-120. https://doi.org/10.1107/s002188989401037x

${ }^{2}$ Cervellino Antonio, Paul Scherrer Institut, Villigen Switzerland, unpublished.

${ }^{3}$ Gozzo Fabia, Excelsus Structural Solutions, Villigen, Switzerland, unpublished.
} 